\title{
Estimating the Tire-Road Friction Coefficient Using Disturbance Observer with Compensator
}

\author{
Ming Liu ${ }^{1,2}$ Xuewen Rong ${ }^{1, *}$ Yibin $\mathrm{Li}^{1}$ and Yanfang Yin $^{2}$ \\ ${ }^{I}$ School of Control Science and Engineering, Shandong University, Jinan, Shandong 250061, China \\ ${ }^{2}$ Department of Electrical Engineering and IT of Shandong University of Science and Technology, Jinan, Shandong \\ 250031, China
}

\begin{abstract}
The tire-road friction coefficient is critical to the performance of mobile robots working outdoors. However, it is difficult to measure using commercially available technology sensors. This paper presents a method of estimating the tire-road friction coefficient using a disturbance observer (DO) with compensator; the chassis speed is not needed. In this paper, the torque produced by the tire-road friction is considered to be the disturbance. The DO with compensator is designed to estimate the tire-road friction coefficient using the dynamic behavior of the vehicle model, the traction torque and the wheel velocity. The simulation result shows that the proposed method can estimate the tire-road friction coefficient accurately under different road conditions and has strong robustness on modeling mismatch and vehicle load changing.
\end{abstract}

Keywords: Disturbance Observer, Vehicle Modeling, Tire-road Friction Coefficient Estimation.

\section{INTRODUCTION}

When a wheeled mobile robot works in various complicated road environments, the tires are the only contact between the robot and the road. Therefore, the friction between the tire and the road is critical to the automatic control of robot's stability. And not only that, intelligent vehicles are equipped with an electronic stability control (ESC) system and anti-lock braking system (ABS) to improve the stability, security and handling performance. In fact, these systems can not work properly without tire-road friction. However, the tire-road friction is influenced by un-controllable environmental characteristics, such as temperature, wear, normal force, tire pressure, and other factors. So it is very difficult to measure directly [1].

In recent years many researches have been focusing on the estimation of tire-road friction coefficient. The approaches can be categorized into cause-based and effect-based methods [2-4]. Cause-based methods detect materials covering road surfaces directly by using such sensors as laser, ultrasonic, microwave and so on. The effect-based methods utilize vehicle and tire dynamic models and other relationships between them to estimate the tire-road friction.

The methods in the first category can estimate the friction coefficient of the road ahead of time which can be beneficial. However, they usually do not measure other factors affecting friction, such as tire conditions; the cost of these sensors do

\footnotetext{
*Address correspondence to this author at the School of Control Science and Engineering, Shandong University, Jinan, Shandong 250061, China; E-mail: scrobot@163.com
}

not allow an industrial implementation. So many approaches using vehicle and tire dynamic models have already been presented to estimate the friction coefficient. Ray [5] uses an Extended Kalman Filter (EKF) to estimate the tire-road forces based on a nine degree of freedom vehicle model. Gerard [6] uses an EKF and an Unscented Kalman Filter (UKF) to estimate tire-road forces and friction coefficient. Belgacem et al. [7] present a method to estimate the longitudinal tireroad force of the vehicle using FOSMO (First Order Slide Model Observer) \& SOSMO(Second Order Slide Model Observer). Liu and Peng [8] use the Luenberger Observer for a 2 DOF (Degree Of Freedom) vehicle model to estimate the friction coefficient. C. Ahn et al. [9] presents a method to estimate road friction coefficient by using robust nonlinear observer. K. Li In [10], three different observers are developed for the estimation of tire forces.

In this paper, we combine the quarter vehicle model and the Magic Formula [11] together to get the model of the system. In order to estimate the tire-road friction coefficient, we consider the torque produced by tire-road friction as interference torque and design a new disturbance observer to estimate it. After we estimate the torque produced by tire-road friction, we can get tire-road friction coefficient easily. The main idea of this approach is to estimate the tire-road friction using observer with compensator not using sensors to measure the chassis speed. In fact, it is very difficult to measure the chassis speed accurately even if it can be measured by using GPS (Global Positioning System). Compared with traditional observer, this new method can decrease the estimating error caused by the error between the model and the actual controlled object. 


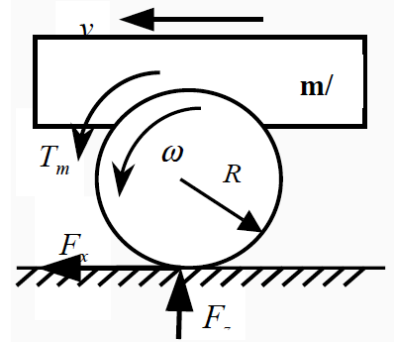

Fig. (1). Simplified quarter vehicle model.

\section{THE SIMPLIFIED VEHICLE MODEL}

\subsection{The Quarter Vehicle Model}

Many studies deal with vehicle modeling in the literature $[12,13]$. These are complex and nonlinear systems and some of the models are difficult to obtain and use in practice. In this paper, we utilize the simplified quarter car model [14] [15] widely reported in the literature. This model retains the main characteristics of the longitudinal dynamics. Assuming the road surface is horizontal, ignoring the rotating resistance, air resistance and lateral motion, the simplified quarter vehicle model is shown as Fig. (1).

According to Newton's law, the mathematical model of the quarter vehicle can be described by

$$
\left.\begin{array}{l}
T_{m}-F_{x} R=J \dot{\omega} \\
F_{x}=F_{z} \mu \\
F_{z}=(m / 4) g \\
F_{x}=(m / 4) \dot{v}
\end{array}\right\}
$$

Where $T_{m}$ is the motor torque, $N \cdot m$,

$F_{x}$ is the longitudinal tire-road friction, $N$,

$R$ is the wheel radius, $m$,

$J$ is the wheel rotational inertia, $\mathrm{kg} \cdot \mathrm{m}^{2}$,

$\omega$ is the wheel angular velocity, $\mathrm{rad} / \mathrm{s}$,

$\mu$ is the tire-road friction coefficient,

$m$ is the vehicle mass, $\mathrm{kg}$,

$g$ is the gravitational acceleration constant, $\mathrm{m} / \mathrm{s}^{2}$,

$v$ is the longitudinal velocity of the vehicle, $m / s$.

\subsection{The Tire Model}

A tire model describes the relationship between tire parameters, tire dynamic states and tire forces. Many tire models have been proposed; the Dugoff Tire Model [16, 17], the Brush Model [18] and the Magic Formula [19] are three widely used tire models. In this paper, the Magic Formula is selected as the tire model. The major drawback of the Dugoff and Brush models is that they both use separate

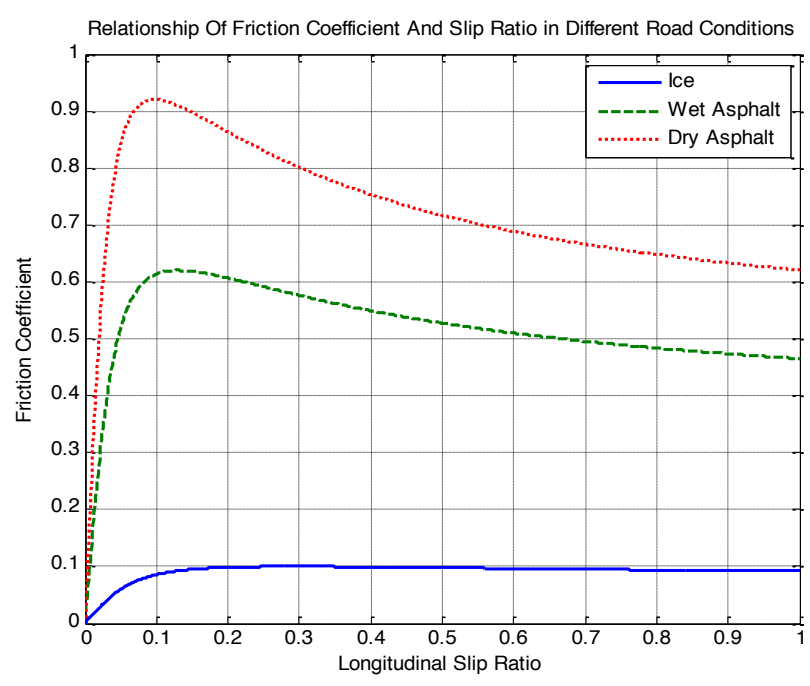

Fig. (2). Relationship of friction coefficient $\mu$ and slip ratio $\lambda$ in different road conditions.

equations for the linear and non-linear regions. However, the size of these regions is a function of $\lambda$. Therefore the algorithm cannot be sure which equation to use when the goal is to estimate $\lambda$. The major advantage of the Magic Formula is that it is a single equation and can achieve high accuracy.

The basic form of Magic Formula for slip ratio and friction coefficient is:

$\mu=D \sin \left(C \tan ^{-1}\left(B \lambda-E\left(B \lambda-\tan ^{-1}(B \lambda)\right)\right)\right.$

Where $\mu$ is the tire-road friction coefficient, $\lambda$ is the longitudinal slip ratio defined as

$\lambda=\frac{R \omega-v}{R \omega}$

It can be seen that equation (2) contains a set of parameters: $B, C, D$ and $E$. These parameters are dependent on the physical properties of the tire and the dynamic state of the vehicle. In equation (2), $D$ represents the peak coefficient, $C$ represents the shape coefficient and influences the shape of the curve, $B$ is the stiffness coefficient, $E$ is the coefficient of curvature.

Fig. (2) shows the $\mu-\lambda$ curves for various road conditions, dry asphalt, wet asphalt and ice.

\subsection{The Simplified Model with Magic Formula}

Combine the two model, we can have the simplified model with Magic Formula as shown in Fig. (3).

In this model, $T_{d}=F_{x} R$ is considered to be the external interference torque and is estimated from the observed disturbance. Then according the simplified model with Magic Formula, we can estimate the tire-road friction coefficient. 


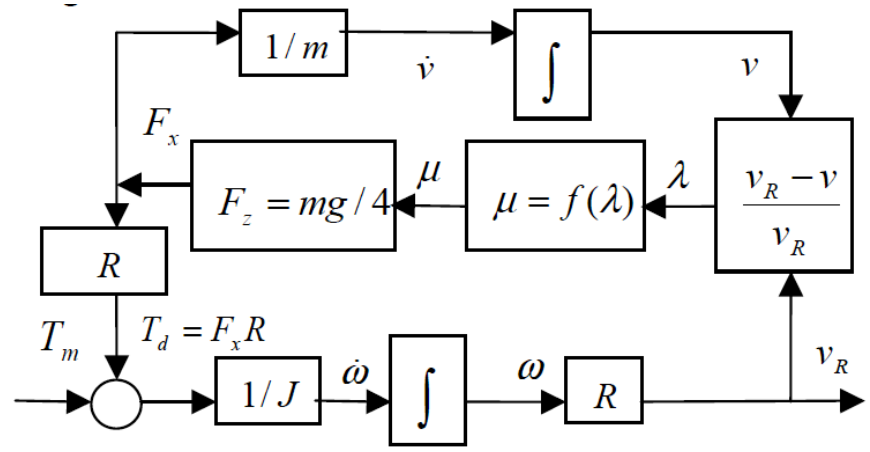

Fig. (3). The simplified model with Magic Formula.

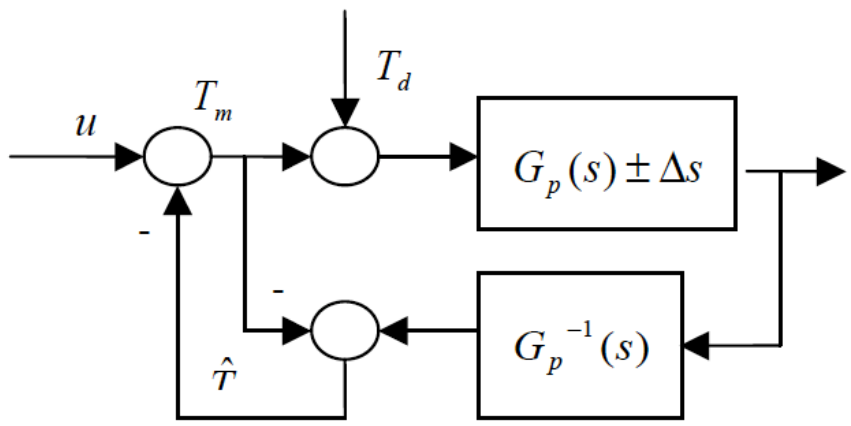

Fig. (4). The basic structure of the disturbance observer.

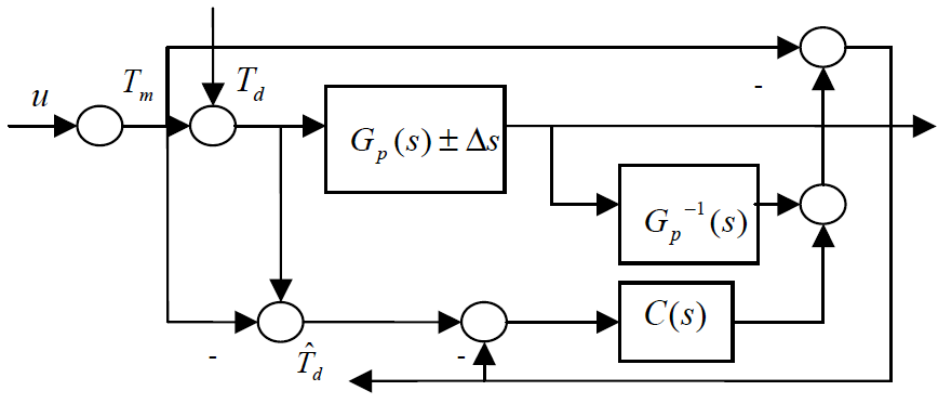

Fig. (5). Block of the disturbance observer with compensator.

\section{ESTIMATING THE TIRE-ROAD FRICTION CO- EFFICIENT BASED ON DISTURBANCE OBSERVER}

\subsection{Design of Disturbance Observer}

Generally in motion control systems, the disturbance observer is used to estimate the equivalent disturbance caused by external interference torque and a parametric variation of the model for compensating the influence of the disturbance. The basic structure is shown as Fig. (4).

Where $\Delta s$ represents the error between the model and the actual controlled object.

According Fig. (3), we can easily obtain

$\hat{T}_{d}=\left[\left(T_{m}+T_{d}\right)\left(G_{p}(s) \pm \Delta s\right)\right] G_{p}^{-1}(s)-T_{m}$
Using equation (4), the disturbance observer can estimate the external interference torque sufficiently if $|\Delta s| \rightarrow 0$. However, there must be modeling error between the model $G_{p}(s)$ and the actual controlled object. That means $\hat{T}_{d} \neq T_{d}$ if $G_{p}(s)$ is replaced by the actual controlled object. And the estimation accuracy of the observer will be influenced by the modeling error.

In order to decrease the influence caused by the modeling error, a new structure of the disturbance observer with modeling error compensation is presented. The main idea is shown as Fig. (5).

According Fig. (4), we can obtain 


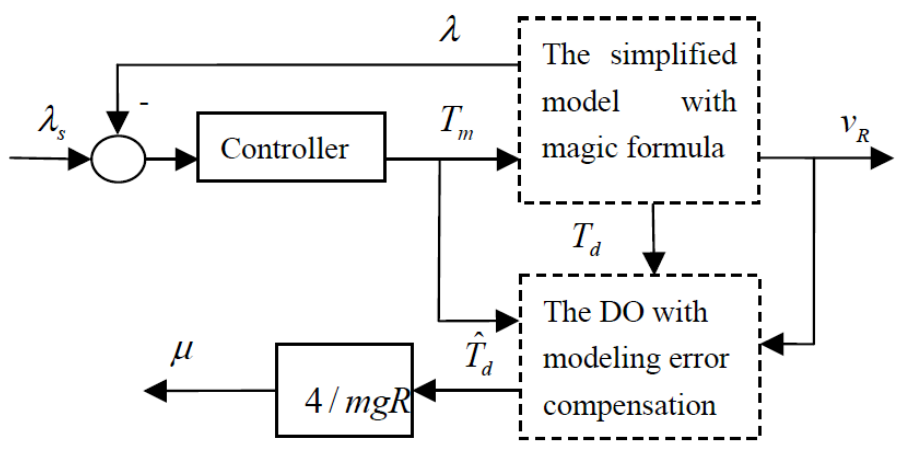

Fig. (6). Block of the traction control system for one wheel.

$\left(T_{m}+T_{d}\right)\left[G_{p}(s) \pm \Delta s\right] G_{p}^{-1}(s)+\left(T_{d}-\hat{T}_{d}\right) C(s)-T_{m}=\hat{T}_{d}$

In equation (5), $C(s)$ is the compensator.

Equation (5) can be written

$\hat{T}_{d}=\frac{\left(T_{m}+T_{d}\right)\left[G_{p}(s) \pm \Delta s\right] G_{p}^{-1}(s)+T_{d} C(s)-T_{m}}{1+C(s)}$

If $|\Delta s| \rightarrow 0$, equation (5) can be written $\hat{T}_{d}=T_{d}$.

Equation (6) can be written

$\hat{T}_{d}=T_{d} \pm \frac{\left(T_{m}+T_{d}\right) \Delta s G_{p}^{-1}(s)}{1+C(s)}$

Consider equation (7): if $\Delta T_{d}=\left|\frac{\left(T_{m}+T_{d}\right) \Delta s G_{p}^{-1}(s)}{1+C(s)}\right| \rightarrow 0$, we still have $\hat{T}_{d} \approx T_{d}$ even if $|\Delta s| \neq 0$ (there must be modeling error).

Moreover, equation (7) can be reconstructed into a compensation problem. It is obvious that the compensator $C(s)$ offers a mechanism to minimize the modeling error caused by $\Delta s$.

In order to minimize the estimation error of $T_{d}, C(s)$ should meet inequality (8).

$|1+C(s)| \gg\left|\left(T_{m}+T_{d}\right) \Delta s G_{p}^{-1}(s)\right|$

In practice, $C(s)$ should meet inequality (9) at least.

$|1+C(s)|>10\left|\left(T_{m}+T_{d}\right) \Delta s G_{p}^{-1}(s)\right|$

Obviously, this disturbance observer has stronger robustness to modeling error than the first one and the robustness is mainly reliant on the compensator. In this paper, we use the classical PID (Proportion Integration Differentiation) compensator. Of course, other advanced compensators can be used.

\section{SIMULATIONS}

In order to verify the validity of the disturbance observer, a simulated traction control system was designed. Fig. (6) shows the basic structure of the simulated traction control system.

The main parameters of the quarter vehicle simulation are shown in Table $\mathbf{1}$. The compensator is $C(s)=20+5 / s$. Where $C(s)$ can meet inequality (9).

Table 1. Specification of the quarter vehicle.

\begin{tabular}{|c|c|}
\hline Total Mass & $240 \mathrm{~kg}$ \\
\hline \hline Wheel Inertia & $0.5 \mathrm{~kg} \cdot \mathrm{m}^{2}$ \\
\hline Wheel Radius & $0.2 \mathrm{~m}$ \\
\hline Initial Chassis Velocity & $36 \mathrm{~km} / \mathrm{h}$ \\
\hline Gravitational Acceleration & $9.8 \mathrm{~m} / \mathrm{s}^{2}$ \\
\hline
\end{tabular}

\subsection{Simulation Based on Standard Road Conditions}

As we all know, the tire-road friction coefficient is closely related to the slip ratio. In order to ensure the tire-road friction, the slip ratio is regarded as the given input in this traction control system.

In fact, the slip ratio is one of the very important indicators in vehicle stability control process. Where the given slip ratio $\lambda_{s}=0.12$, the initial slip ratio $\lambda_{0}=0$, the parameters in different standard road conditions are shown as Table 2 [8]. And the simulation result is shown as Fig. (7).

Table 2. The parameters in different standard road conditions.

\begin{tabular}{|c|c|c|c|c|}
\hline & $\boldsymbol{E}$ & $\boldsymbol{B}$ & $\boldsymbol{C}$ & $\boldsymbol{D}$ \\
\hline \hline Dry Asphalt & 0.6 & 19.25 & 1.65 & 0.92 \\
\hline Wet Asphalt & 0.6 & 15.8 & 1.6 & 0.62 \\
\hline Ice & 0.6 & 9.8 & 1.45 & 0.1 \\
\hline
\end{tabular}

The simulation result shows there is little difference between the estimated coefficient and the actual coefficient and the DO with compensator can estimate the tire-road friction coefficient accurately and quickly. 

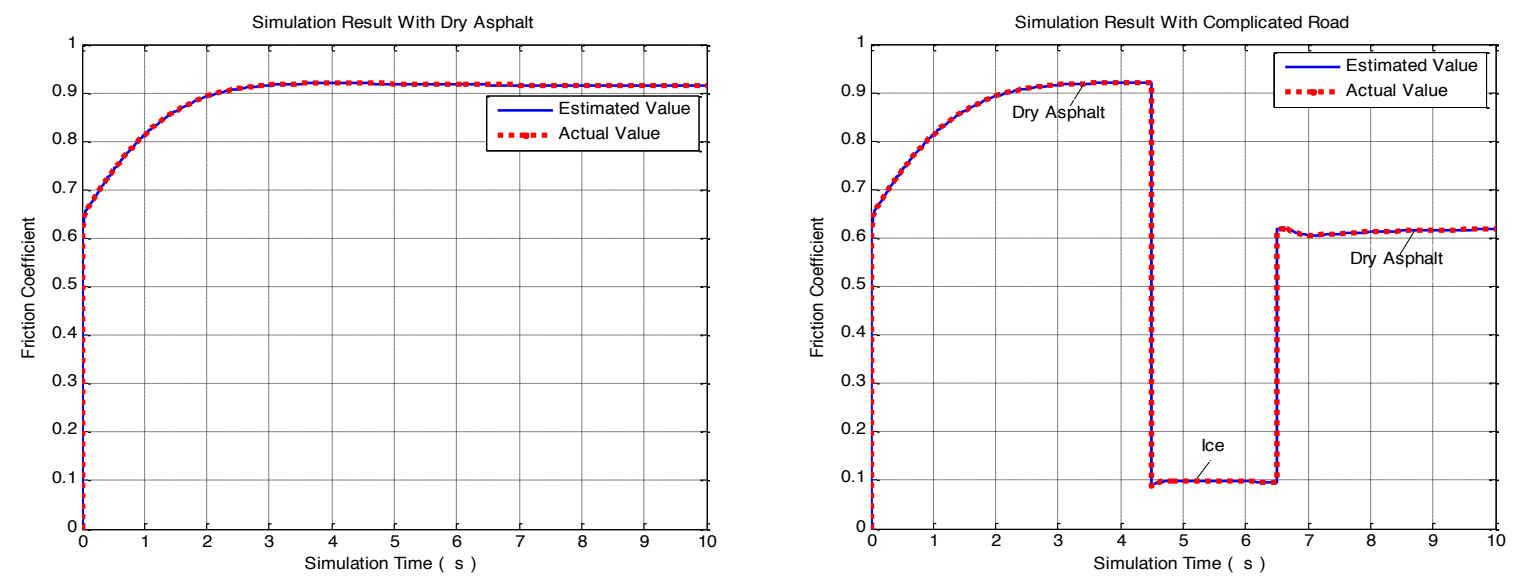

Fig. (7). Simulation result with different road conditions.
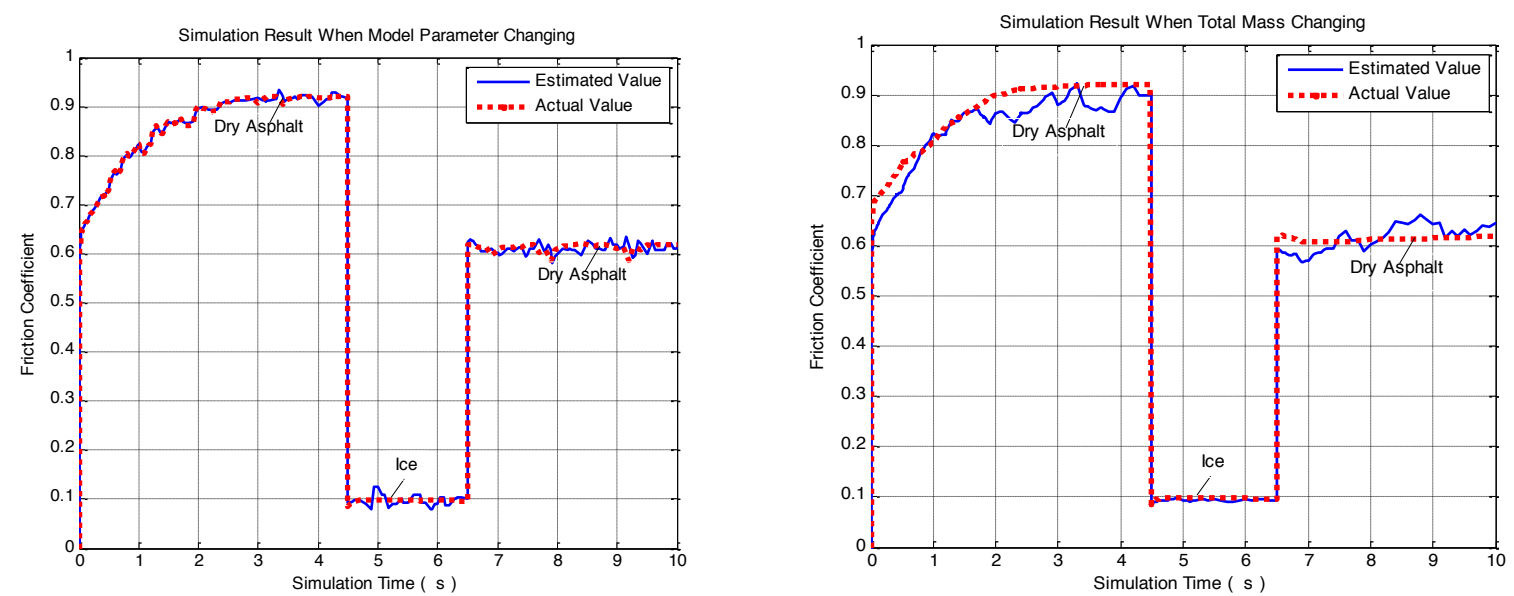

Fig. (8). Simulation result when model parameters and total mass changing.

\subsection{Simulation When Some of the System Parameters Changing}

In fact, we cannot ensure the model can match the disturbance observer completely, that means $|\Delta s| \neq 0$. And load of the vehicle must be changed in practice. That is to say the total mass will be changed. In order to test the robustness of this observer, we simulate the system by changing the model and total mass of this vehicle randomly.

Consider the standard proportionality coefficient as $k$, and total mass of the vehicle as $m$. In the process of simulating, these two parameters will change as follows:

$k^{\prime}=k\{1+0.1[\operatorname{rand}(1)-0.5]\}$

$m^{\prime}=m\{1+0.1[\operatorname{rand}(1)-0.5]\}$

The simulation result is shown as Fig. (8).

The simulation shows that the observer can still estimate the tire-road friction coefficient accurately and quickly and the robustness to model mismatching is better with the load changing. In the process of simulation, $\mathrm{k}$ is the proportionality coefficient of the simplified vehicle model and $\mathrm{m}$ is the total mass of the vehicle. These two parameters are constant when the vehicle is specified.

\subsection{Simulation when Given Slip Ratio Changing}

In practice, the given slip ratio must change. Fig. (9) shows the simulation result.

The simulation result shows whether or not the road condition, the model parameters and the total mass of the vehicle change, the disturbance observer can still work well, even if the slip ratio can not track the given curve.

\section{CONCLUSION}

This paper proposes a method of estimating the tire-road friction coefficient. This method is based on the quarter vehicle dynamic model, the Magic Formula and the disturbance observer with compensator. The simulation results 

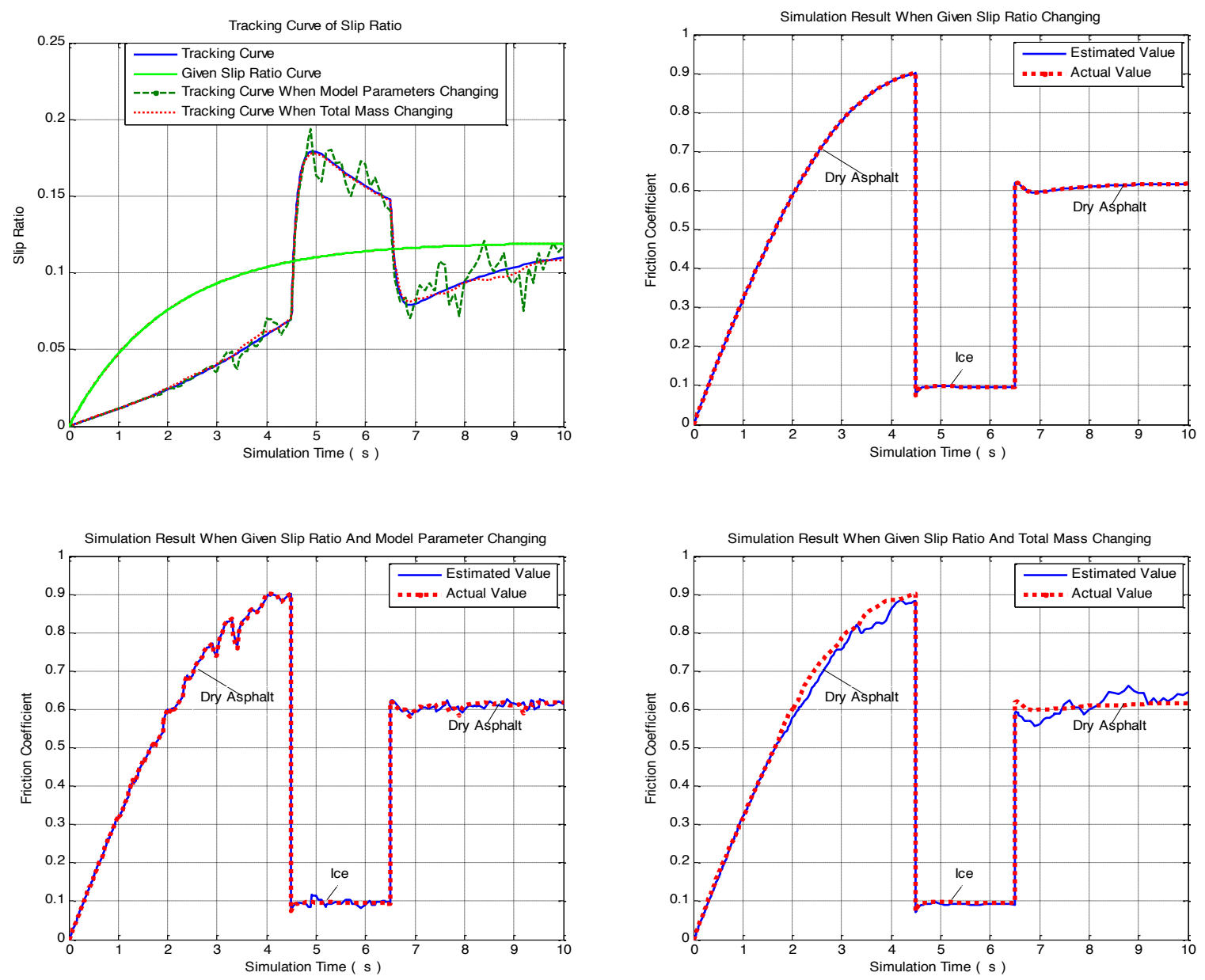

Fig. (9). Simulation result when model given slip ratio, parameters and total mass changing.

show this method works well and has strong robustness to model parameter mismatching and load changing. The choice of the parameters of the compensator is very important. Because it is very difficult to get the models of the vehicle, the choice of the parameters of the compensator is difficult too. For future work, experiments will be performed on real vehicle in different conditions to verify the validity of the disturbance observer and try to find better ways to chose the parameters of the compensator.

\section{CONFLICT OF INTEREST}

The authors declare that there is no conflict of interests regarding the publication of this paper.

\section{ACKNOWLEDGMENTS}

This study is supported by the National Natural Science Foundation of China $(61233014,61305130)$ and the Fundamental Research Funds of Shandong University (2014 JC037).

\section{REFERENCES}

L. R. Ray, "Nonlinear tire force estimation and road friction identification: simulation and experiments", Automatica, vol.33, pp.1819-1833, 1997.
[2] Y. Sasada, "Development of the road surface condition sensing system", International Conference on Intelligent Transportation Systems, Tokyo, 1999:14-19.

[3] M. Yamada, K. Ueda, I. Horiba, S. Tsugawa, and S. Yamamoto, "Road surface condition detection technique based on image taken by camera attached to vehicle rearview mirror", Review of Automotive Engineering, vol. 26, pp. 163-168, 2005.

[4] Y. Sato, A. D. Kobay, I. Kageyama, K. Watanabe, Y. Kuriyagawa, and Y. Kuriyagawa, "Study on recognition method for road friction condition", JSAE Transaction, vol. 38, pp. 51-56, 2007.

[5] L. R. Ray, "Nonlinear state and tire force estimation for advanced vehicle control", IEEE Transactions on Control Systems Technology, vol.3, no.1, pp. 117-124, 1995.

[6] M. Gerard, Tire-road Friction Estimation Using Slip-Based Observers, Master Thesis, Lund University, Sweden, 2006.

[7] B. Jaballah, N. Kouider M'SIRDI, A. Naamane, and H. Messaoud, "Estimation of vehicle longitudinal tire force with FOSMO \& SOSMO", International Journal of Science and Techniques of Automatic Control \& Computer Engineering, vol.5, no.1, pp. 1516$1531,2011$.

[8] C. Liu, and H. Peng, "Road friction coefficient estimation for vehicle path prediction", Vehicle System Dynamics, vol.25, pp. 413425,1996.

[9] C. Ahn, H. Peng, and H. E. Tseng, "Robust nonlinear observer to estimate road friction coefficient and tire slip angle," $10^{\text {th }}$ International Symposium on Advanced Vehicle Control, Loughborough, UK, 2010.

[10] R. Rajamani, G. Phanomchoeng, D. Piyabongkarn, and J. Y. Lew, "Algorithms for real-time estimation of individual wheel tire-road friction coefficients," IEEE/ASME Transactions on Mechatronics, vol. 17, no. 6, pp.1183-1195, 2012.

[11] B. Li, H. Du, and W. Li, "A novel cost effective method for vehicle tire-road friction coefficient estimation," IEEE/ASME International 
Conference on Advanced Intelligent Mechatronics (AIM), 2013, pp. 1528-1533.

[12] H. Lee and M. Tomizuka, "Adaptive vehicle traction force control for intelligent vehicle highway systems (IVHSs)," IEEE Transactions on Industrial Electronics, vol. 50, no. 1, 2003.

[13] N. K. M'Sirdi, A. Rabhi, A. Zbiri, and Y. Delanne, "VRIM: vehicle road interaction modelling for estimation of contact forces", In: TMVDA 04. $3^{\text {rd }}$ Int. Tyre Colloquium Tyre Models for Vehicle Dynamics Analysis, August 30-31, University of Technology Vienna, Austria, 2004.

[14] C. Canudasde Wit, P. Tsiotras, E. Velenis, M. Basset, and G. Gissinger, "Dynamic friction models for road/tire longitudinal interaction”, Vehicle System Dynamics, vol. 39, no. 3, pp. 189-226, 2003.
[15] U. Kiencke, and L, Nielsen, Automotive Control Systems for Engine, Driveline and Vehicle. Springer-Verlag, 2005.

[16] H. Dugoff, P. Fancher, and L. Segel ,"Tire performance characteristics affecting vehicle response to steering and braking control inputs", Control Systems Technology, vol. 460, no. 17, 1969.

[17] R. Guntur, and S. Sankar, "A friction circle concept for Dugoff's tyre friction model”, International Journal of Vehicle Design, vol. 1, no. 4, pp. 373-377, 1980 .

[18] H. Pacejka, Tyre and Vehicle Dynamics, SAE International, 2005.

[19] H. Pacejka, and R. Sharp, "Shear force development by pneumatic tyres in steady state conditions: a review of modelling aspects", Vehicle System Dynamics, vol. 20, no. 3-4,pp. 121-175, 1991.

(C) Liu et al.; Licensee Bentham Open.

This is an open access article licensed under the terms of the Creative Commons Attribution Non-Commercial License (http://creativecommons.org/licenses/by-nc/4.0/) which permits unrestricted, non-commercial use, distribution and reproduction in any medium, provided the work is properly cited. 